Scientia Agropecuaria

\title{
Respuesta del maíz (Zea mays L) a la inoculación con Azotobacter sp y Burkholderia sp a dosis reducida de fertilizante nitrogenado
}

\section{Maize responds to Azotobacter sp and Burkholderia sp inoculation at reduced dose of nitrogen fertilizer}

Juan Manuel Sánchez-Yáñez ${ }^{1, *}$, Irma Yatziri López Ayala ${ }^{1}$, Javier Villegas Moreno $^{2}$, Noé Manuel Montaño Arias ${ }^{3}$

${ }^{1}$ Laboratorio de Microbiología Ambiental, Instituto de Investigaciones Químico Biológicas, Ed-B3 C.U., Universidad Michoacana de San Nicolás de Hidalgo, Francisco J. Mujica S/N, Col. Felicitas del Rio C.P. 58000, Morelia, Mich., México.

2 Microorganismo-suelo-planta. Instituto de Investigaciones Químico Biológicas, Ed-B3 C.U., Universidad Michoacana de San Nicolás de Hidalgo, Francisco J. Mujica S/N, Col. Felicitas del Rio C.P. 58000, Morelia, Mich., México.

${ }^{3}$ Departamento de Biología (Botánica), División de Ciencias Biológicas y de la Salud, Universidad Autónoma Metropolitana Unidad Iztapalapa, México. DF, México.

Recibido 17 noviembre 2013. Aceptado 18 marzo 2014.

\section{Resumen}

La respuesta positiva del maíz a la inoculación con bacterias promotoras de crecimiento vegetal (BPCV) como los géneros bacterianos: Azotobacter sp. y Burkholderia sp. endofita es una alternativa para la reducción y optimización de la dosis del fertilizante nitrogenado $(\mathrm{FN})$ como el nitrato de amonio $\left(\mathrm{NH}_{4} \mathrm{NO}_{3}\right)$, recomendado para esta gramínea, sin afectar negativamente su crecimiento. El objetivo de este trabajo fue analizar la respuesta del maíz a la inoculación con Azotobacter sp. y Burkholderia sp. a la dosis 50\% del FN. El experimento se realizó bajo un diseño experimental de bloques al azar, mediante las variables respuesta para analizar el efecto de BPCV en el maíz: porcentaje de germinación (\%); la fenotípia aérea y radical; y la biomasa: peso fresco aéreo (PFA) y radical (PFR); el peso seco aéreo (PSA) y radical (PSR). Los resultados indicaron un efecto positivo de ambos géneros de BPCV en el maíz desde su germinación, a nivel de plántula y en floración. En el maíz coinoculado se registró un PSR de 7,03 g, valor estadísticamente significativo comparado con los 2,60 g de PSR del maíz sin inocular, alimentado con la dosis de FN recomendado, empleado como control relativo. Lo anterior sugiere una interacción sinérgica de Azotobacter sp. y Burkhoderia sp. en la síntesis de sustancias promotoras de crecimiento vegetal (SPCV) en maíz para optimizar la dosis reducida del FN y permitir su sano crecimiento.

Palabras clave: colonización, rizósfera, absorción radical.

\begin{abstract}
The positive maize response to inoculation with plant growth promoting bacteria (PGPB) as Azotobacter sp and Burkholderia sp an endophytic type, are an alternative to reduced and optimize nitrogen fertilizer (NF) dose, recommended for this plant, without adversely affect its growth. The aim of this study was to analyze maize respond to inoculation with Azotobacter sp and Burkholderia sp at the dose 50\% of FN. Used an experimental design of randomized blocks. By response variables: percent germination (\%), the shoot and root phenology: plant height (PH), root length (RL) and biomass: shoot fresh weight (SFW) and root fresh weight (RFW), the shoot dry weight (SDW) and root dry weight (RDW). The results indicated a positive maize respond to PGPB inoculation at germination, seedling and flowering level, reached a RDW of $7.03 \mathrm{~g}$, statistically significant value compared with $2.60 \mathrm{~g}$ of RDW non inoculated maize feed with NF dose recommended regard as relative control (RC). This suggests a synergistic interaction among these PGPB in synthesis of plant growth promoting substances (PGPS) on maize, to optimize the reduced NF dose.
\end{abstract}

Keywords: colonization, rhizosphere effect, root absorption.

\footnotetext{
* Autor para correspondencia

E-mail: syanez@umich.mx (J. Sánchez-Yáñez)
} 


\section{Introducción}

El Zea mays L "maíz" es el cereal más importante después del Tricticum aeuestium "trigo" en el comercio mundial. En México el maíz es materia prima para consumo humano, industrial y forraje para animales. Este cereal demanda fertilizante nitrogenado (FN), cuya aplicación en exceso causa la pérdida de productividad del suelo y contaminación ambiental; una alternativa para evitar la hiperfertilización del FN en el maíz, es la inoculación de sus semillas con géneros de bacterias promotoras de crecimiento vegetal (BPCV) del tipo: Azotobacter sp. y Burkholderia sp. endofita (Aguirre-Medina et al., 2009; García-Reyna et al., 2000), que optimizan la dosis reducida del $\mathrm{FN}$, sin afectar negativamente su crecimiento (Armenta et al., 2010). Mientras que Mora y Toro (2007) reportaron la respuesta positiva del maíz a Burkholderia cepacia endófita para optimizar el FN aplicado a dosis reducida al $50 \%$, con un efecto positivo en su crecimiento. Aguirre-Medina et al. (2009) demostraron la acción benéfica de la inoculación individual y en mezcla de $B$. phytofirman y Pseudomonas putida en maíz, al mejorar su funcionamiento radical en la absorción del FN. Al igual que Hernández-Rodríguez et al. (2010), que investigaron que $B$. cepacia colonizo el tejido vegetal radical del maíz e indujo un crecimiento saludable. En ese sentido Piromyou et al. (2011) probaron que Azotobacter sp. al invadir externamente la raíz del maíz, lo favoreció con la síntesis de sustancias promotoras de crecimiento vegetal (SPCV).

Con base en lo anterior, el objetivo de esta investigación fue analizar la respuesta del maíz a la inoculación con Azotobacter sp. y Burkholderia sp. endofita a dosis reducida al $50 \%$ del FN.

\section{Material y métodos}

El experimento se realizó en un suelo latéritico sódico pobre en materia orgánica y Nitrógeno $(\mathrm{N})$ mineral, que se solarizo (García-Reyna et al., 2001). La semilla de maíz se inoculó como se describe en García-González et al., 2005, con los géneros de BPCV que se aislaron de Leucena sp. e identificaron como Azotobacter sp. y Burkholderia sp. ambas pertenecientes a la colección del laboratorio de Microbiología Ambiental del IIQB-UMSNH, Morelia, Mich, México (Sánchez-Yáñez, 2007). La semilla de maíz se inoculó con Azotobacter sp. y Burkholderia sp. en jarra de Leonard y ahí se alimentaron con el $\mathrm{FN}$ en forma de $\mathrm{NH}_{4} \mathrm{NO}_{3}$ (nitrato de amonio) (GarcíaReyna et al., 2000). En la tabla 1, se muestra el diseño experimental empleado para analizar la respuesta del maíz a la inoculación con Azotobacter sp. $y$ Burkholderia sp. a dosis reducida del $50 \%$ del FN con las variables respuesta: porcentaje (\%) de germinación de la semilla, mientras que a nivel plántula y en floración; la fenotípia: la altura de planta (AP), la longitud de raíz (LR); y para la biomasa: el peso fresco aéreo (PFA) y radical (PFR), luego ambas partes del maíz se secaron en horno a $40^{\circ} \mathrm{C} / 48 \mathrm{~h}$ y se obtuvo el peso seco de la parte aérea (PSA) y radical (PSR). La validación de los datos experimentales se realizó por Tukey (García et al., 2001).

\section{Resultados y discusión}

En la tabla 2 se muestra el $\%$ de germinación de la semilla de maíz, 11 días después de la siembra, la semilla inoculada con Burkholderia sp. endófita alcanzó un $95 \%$ de emergencia, mientras que la semilla de maíz tratada con éste género de BPCV y Azotobacter sp. fue de $95 \%$ a la dosis $50 \%$ de $\mathrm{FN}$, estos valores fueron estadísticamente significativos superiores comparados con el $85 \%$ de la germinación del maíz sin inocular a la dosis $100 \%$ del FN (CR). 


\section{Tabla 1}

Diseño experimental para medir la respuesta de maíz a la inoculación con Azotobacter sp y Burkholderia sp. endofita a dosis reducida de fertilizante nitrogenado

\begin{tabular}{cccc}
\hline Tratamiento/maíz & Azotobacter $\mathrm{sp}$. & Burkholderia sp. & $\begin{array}{c}\text { Fertilizante nitrogenado } \\
\left(\mathrm{NH}_{4} \mathrm{NO}_{3}\right)\end{array}$ \\
\hline 1 Control absoluto & - & - & $100 \% \overline{(10 \mathrm{~g} / \mathrm{L})}$ \\
2 Control relativo & - & - & $50 \%(5 \mathrm{~g} / \mathrm{L})$ \\
3 & - & + & “ \\
4 & - & + & " \\
5 & + & - & “ \\
6 & + & - & " \\
7 & + & + & + \\
\hline
\end{tabular}

$*+=$ aplicado, $-=$ No aplicado.

Lo anterior sugiere que tanto Azotobacter sp. y Burkholderia sp. convirtieron los exudados de la espermosfera de la semilla del maíz en SPCV, para terminar con la latencia del embrión y acelerar su velocidad de germinación (Arellano et al., 2008; Martínez et al., 2011), al igual que el maíz que germino en un $90 \%$ tratado individualmente con Azotobacter sp. lo que de acuerdo con la literatura, sugiere que existen compuestos orgánicos del tipo aminoácidos como el triptófano que la semilla de la gramínea exuda y que ambos géneros de BPCV transformaron en SPCV (García-Reyna et al., 2001; GarcíaGonzález et al., 2005; Wong-Villareal y Caballero-Mellado, 2010), lo que indujo un porcentaje de germinación superior al del maíz tratado solo con el FN al $100 \%$ usado como CR.

\section{Tabla 2}

Respuesta de la semilla de maíz a la inoculación con Azotobacter sp. y Burkholderia sp y la mezcla de ambas en su germinación, 11 días después de la siembra

\begin{tabular}{ll}
\hline Tratamiento/maíz & $\begin{array}{c}\text { Germinación } \\
(\%)\end{array}$ \\
\hline Agua (control absoluto) & $75^{\mathrm{d}^{*}}$ \\
\hline $\begin{array}{l}\text { Fertilizante nitrogenado } \\
100 \% \text { (control relativo) }\end{array}$ & $85^{\mathrm{c}}$ \\
\hline Azotobacter $\mathrm{sp}$ & $90^{\mathrm{b}}$ \\
\hline Burkholderia $\mathrm{sp}$ & $95^{\mathrm{a}}$ \\
\hline $\begin{array}{l}\text { Azotobacter } \mathrm{sp} y \\
\text { Burkholderia } \text { sp }\end{array}$ & $95^{\mathrm{a}}$ \\
\hline $\begin{array}{l}\text { * Valores con letras distintas } \\
\text { estadística }(\mathrm{p}<0,05) \text { según Tukey. }\end{array}$ &
\end{tabular}

En la tabla 3 se muestra la respuesta positiva del maíz a los 32 días después de la siembra, inoculado con Azotobacter sp. que causó un PSA de 2,03 g, ya que este género de BPCV tiene capacidad de reconocer y convertir algunos exudados radicales del maíz en SPCV, para una rápida y mejor absorción del $\mathrm{FN}$ reducido al 50\% (Reyes y Valery, 2007)

En tanto que la gramínea inoculada con Azotobacter sp. y Burkholderia sp. registro 2,56 g de PSA, dado que sus SPCV aceleran y aumentan la capacidad de la raíz para absorber el FN reducido al 50\% y otros minerales del suelo y ello le permite al maíz crecer rápido y sanamente (Armenta et al., 2010; HernándezRodríguez et al., 2010; Martínez et al., 2011).

\section{Tabla 3}

A plántula respuesta del maíz a la inoculación con Azotobacter sp. y Burkholderia sp. en su biomasa 32 días después de la siembra

\begin{tabular}{llc}
\hline \multirow{2}{*}{ Tratamientos/maíz } & \multicolumn{2}{c}{ Peso seco $(\mathrm{g})$} \\
\cline { 2 - 3 } & Aéreo & Radical \\
\hline Agua (control absoluto) & $0,84^{\mathrm{b} *}$ & $0,53^{\mathrm{d}}$ \\
\hline $\begin{array}{l}\text { Fertilizante nitrogenado } \\
100 \% \text { (control relativo) }\end{array}$ & $0,84^{\mathrm{b}}$ & $0,60^{\mathrm{c}}$ \\
\hline Azotobacter $\mathrm{sp}$. & $2,03^{\mathrm{a}}$ & $0,59^{\mathrm{c}}$ \\
\hline Burkholderia sp & $0,98^{\mathrm{b}}$ & $0,63^{\mathrm{b}}$ \\
\hline $\begin{array}{l}\text { Azotobacter } \mathrm{sp} . y \\
\text { Burkholderia } \text { sp. }\end{array}$ & $2,56^{\mathrm{a}}$ & $0,80^{\mathrm{a}}$ \\
\hline $\begin{array}{l}\text { *Valores con diferentes letras con } \\
\text { estadística }(\mathrm{p}<0,05) \text { diferencia }\end{array}$ & &
\end{tabular}


En ambos casos estos valores fueron estadísticamente diferentes y significativos comparados con los del maíz con $0,84 \mathrm{~g}$ de PSA sin inocular, usado como CR e igual al valor del PSA del maíz inoculado solo con Burkholderia sp. con 0,98 g; lo que sugiere que los dos géneros de BPCV transformaron los exudados radicales del maíz en SPCV (Armenta et al., 2010; Martínez et al., 2011), con lo que hubo una mejora en la absorción del FN a pesar de reducirlo al 50\% (Mora y Toro, 2007) y así un incremento en su PFA (Aguirre-Medina et al., 2009; Hernández-Rodríguez at al., 2010; Piromyou et al., 2011). Lo que también se registró cuando el maíz se inoculó solo con alguno de los dos géneros de BPCV indicados; en cuyo caso los valores del PSA fueron estadísticamente diferentes y significativos comparados con el maíz sin inocular, tratado solo con el FN al $100 \%$ del CR. Mientras que el maíz inoculado con Azotobacter sp. y Burkholderia sp. a la dosis de FN al 50\%, registro un PSR de 0,80 g, lo que sugiere que Azotobacter sp y Burkholderia sp a nivel de la raíz del maíz, convirtieron sus exudados en SPCV, para una mayor formación de raíces secundarias que optimizaron la dosis reducida al 50\% del FN (Arellano et al., 2008; Armenta et al., 2010; Piromyou et al., 2011) en consecuencia hubo un incremento en la longitud y número de raíces secundarias de la gramínea (Wong-Villareal y CaballeroMellado, 2010), al igual que el maíz tratado, solo con alguno de los dos géneros de BPCV y cuyos valores de PSR en ese maíz fueron estadísticamente diferentes $\mathrm{y}$ significativos, en especial el maíz tratado con Burkholderia sp. con 0,63 g; comparado con el valor del PSR del maíz con $0,60 \mathrm{~g}$ y de su homólogo sin inocular alimentado con el FN al $100 \%$ usado como CR, igual al valor de $0,59 \mathrm{~g}$ del PSR del maíz tratado únicamente con Azotobacter sp. lo que evidentemente mostró que este género de BPCV aumento la actividad radical para mejorar $y$ optimizar la absorción radical del FN reducido al 50\% (Aguirre-Medina et al, 2009; Hernández-Rodríguez et al., 2010). En la tabla 4, a floración se muestra la respuesta positiva del maíz a 62 días después de la siembra, inoculado con Burkholderia sp. en su altura de planta (AP) con 113,32 cm, mientras que el maíz tratado con ambas fue de $114,37 \mathrm{~cm}$, lo que sugiere que estos géneros de BPCV transformaron los exudados radicales del maíz en SPCV, las que indujeron un incremento del crecimiento de su tallo (Armenta et al., 2010; Camelo et al., 2011), al igual que lo observado cuando el maíz fue inoculado con uno de los dos géneros de BPCV; este valor fue estadísticamente diferente y significativo comparado con los 99,87 cm de la AP del maíz tratado solo con Azotobacter sp. ello sugiere que este género bacteriano transformó exudados de raíz en SPCV (Arellano et al., 2008; Piromyou et al., 2011) comparado con el valor de la AP del maíz con 95,25 cm de su homólogo sin inocular con el FN al $100 \%$ empleado como CR.

\section{Tabla 4}

A floración respuesta del maíz a la inoculación con Azotobacter sp y Burkholderia sp. y con ambas en su fenotípia 62 días después de la siembra

\begin{tabular}{lcc}
\hline Tratamiento/maíz & $\begin{array}{c}\text { Altura de } \\
\text { planta } \\
(\mathrm{cm})\end{array}$ & $\begin{array}{c}\text { Longitud } \\
\text { radical } \\
(\mathrm{cm})\end{array}$ \\
\hline $\begin{array}{l}\text { Agua (control } \\
\text { absoluto) }\end{array}$ & $92,20^{\mathrm{d}^{*}}$ & $35,85^{\mathrm{e}}$ \\
\hline $\begin{array}{l}\text { Fertilizante } \\
\text { nitrogenado } 100 \% \\
\text { (control relativo) }\end{array}$ & $95,25^{\mathrm{c}}$ & $40,75^{\mathrm{d}}$ \\
\hline Azotobacter $\mathrm{sp}$ & $99,87^{\mathrm{b}}$ & $50,47^{\mathrm{c}}$ \\
\hline Burkholderia $\mathrm{sp}$ & $113,32^{\mathrm{a}}$ & $56,97^{\mathrm{b}}$ \\
\hline $\begin{array}{l}\text { Azotobacter } \mathrm{sp} y \\
\text { Burkholderia } \mathrm{sp}\end{array}$ & $114,37^{\mathrm{a}}$ & $60,01^{\mathrm{a}}$ \\
\hline *Valores con diferentes letras con & diferencia \\
estadística $(\mathrm{p}<0,05)$ según Tukey. & \\
\hline
\end{tabular}

En tanto que el maíz tratado con los dos géneros de BPCV registro 60,01 cm de LR, y la misma gramínea inoculada solo con Burkholderia sp. alcanzó 56,97 cm de 
LR; mientras que el maíz inoculado solo con Azotobacter sp. registró $50,47 \mathrm{~cm}$ de LR, lo anterior indica que este género de BPCV, puede mediante la conversión de exudados radicales del maíz, inducir la formación de un mayor número pelos radicales en la zona de su meristemo, mientras que a la vez Burkholderia sp. endófita convirtió exudados de maíz en SPCV pero en el interior de las raíces del maíz (Armenta et al., 2010; Camelo et al., 2011). La respuesta positiva del maíz a los dos géneros de BPCV de manera individual y en combinación fue estadísticamente diferente y significativa en comparación con el maíz sin inocular con $40,75 \mathrm{~cm}$ de LR con el FN al $100 \%$ utilizado como CR, lo cual muestra que en efecto una adecuada selección de géneros de BPCV; puede ser una herramienta valiosa $\mathrm{p}$ para conservar la fertilidad del suelo en el cultivo del maíz, sin riesgo de contaminar agua superficial y subterránea por el exceso de FN aplicado (AguirreMedina et al., 2009).

En la tabla 5, a floración se muestra la respuesta positiva del maíz a la inoculación con Azotobacter sp. y Burkholderia sp. en su PFA con 47,28 g, a la dosis al 50\% del $\mathrm{FN}$, lo anterior sugiere que ambas BPCV contribuyeron a nivel de la raíz a la síntesis de SPCV, para mejorar su capacidad de absorción radical del $\mathrm{FN}$, lo que en consecuencia causó el incremento en su PFA (Reyes y Valery, 2007; WongVillareal y Caballero-Melado, 2010). Este valor y los registrados en el maíz tratado con Azotobacter sp. o Burkholderia sp. fueron estadísticamente distintos y significativos comparados con los $34,69 \mathrm{~g}$ de PFA del maíz usado como CR sin inocular, alimentado con el FN al $100 \%$, mientras que el maíz inoculado con ambos géneros de BPCV registro un PFR de 28,78 g, lo que sugiere que Azotobacter sp. y Burkholderia sp. estimularon una mayor formación de raíces secundarias, y ello optimizó la absorción del FN reducido al 50\% (Reyes y Valery, 2007; Camelo et al., 2011; Escobar et al., 2011), este valor y el observado cuando el maíz se trató solo con Azotobacter sp. o Burkholderia sp. fuerón estadísticamente diferentes y significativos comparados con el maíz con 17,66 g de PFR sin inocular, alimentado con el $100 \%$ del FN empleado como CR.

\section{Tabla 5}

A floración respuesta del maíz a la inoculación con Azotobacter sp. y Burkholderia sp. y con ambas en su biomasa 62 días después de la siembra

\begin{tabular}{lcc}
\hline \multirow{2}{*}{ Tratamiento/Maíz } & \multicolumn{2}{c}{ Peso fresco $(\mathrm{g})$} \\
\cline { 2 - 3 } & Aéreo & Radical \\
\hline $\begin{array}{l}\text { Agua (control } \\
\text { absoluto) }\end{array}$ & $29,48^{\mathrm{d}^{*}}$ & $12,61^{\mathrm{e}}$ \\
\hline $\begin{array}{l}\text { Fertilizante } \\
\text { nitrogenado (control } \\
\text { relativo) }\end{array}$ & $34,69^{\mathrm{c}}$ & $17,66^{\mathrm{d}}$ \\
\hline Azotobacter $\mathrm{sp}$ & $41,30^{\mathrm{b}}$ & $21,28^{\mathrm{c}}$ \\
\hline Burkholderia $\mathrm{sp}$ & $42,93^{\mathrm{b}}$ & $21,56^{\mathrm{b}}$ \\
\hline $\begin{array}{l}\text { Azotobacter } \mathrm{sp} y \\
\text { Burkholderia } \mathrm{sp}\end{array}$ & $47,28^{\mathrm{a}}$ & $28,78^{\mathrm{a}^{*}}$ \\
\hline $\begin{array}{l}\text { *Valores con diferentes letras con } \\
\text { estadística (p<0,05) según Tukey. }\end{array}$ & \\
\hline
\end{tabular}

En la tabla 6, a floración se muestra la respuesta del maíz a la inoculación mixta en su PSA con 10,35 g, mientras su homólogo tratado solo con Burkholderia sp. alcanzó 9,99 g de PSA y el maíz inoculado únicamente con Azotobacter sp. alcanzó un PSA de 8,54 g, lo que indica que estas BPCV individualmente $\mathrm{y}$ en combinación transformaron los exudados de la raíz del maíz en SPCV; y así optimizaron la absorción del FN, con el consecuente aumento en su biomasa (Wong-Villareal y Caballero-Mellado, 2010; Hernández-Rodríguez et al., 2010), en ambos casos estos valores fueron estadísticamente diferentes y significativos comparados con el maíz con un PSA de $6,28 \mathrm{~g}$ sin inocular con el FN al $100 \%$ usado como CR. El maíz tratado con los dos géneros de BPCV alcanzó un PSR de 7,03 g, lo que indica que Azotobacter sp. y Burkholderia sp. transformaron los exudados radicales del maíz en SPCV complementarias, por lo que derivado de esta acción conjunta, hubo una mayor 
proliferación de pelos radicales para la optimización del FN reducido al $50 \%$ (García-Reyna et al., 2000; GarcíaGonzález et al., 2005); mientras que lo registrado cuando el maíz fue tratado solo con alguno de los dos géneros de BPCV, los valores del PSR fueron estadísticamente diferentes y significativos en especial con Burkholderia sp. con 6,00 $\mathrm{g}$ de PSR y en el maíz inoculado solo con Azotobacter sp. con 5,78 g de PSR, ya que estos valores fueron estadísticamente distintos y significativos comparados con los 2,60 g PSR del maíz sin inocular, alimentado con el FN al $100 \%$ usado como CR.

\section{Tabla 6}

A floración respuesta del maíz a la inoculación con Azotobacter sp y Burkholderia sp y la mezcla de ambas en su biomasa a 62 días después de la siembra

\begin{tabular}{lcc}
\hline \multirow{2}{*}{ Tratamiento/maíz } & \multicolumn{2}{c}{ Peso seco $(\mathrm{g})$} \\
\cline { 2 - 3 } & Aéreo & Radical \\
\hline Agua (control absoluto) & $4,67^{\mathrm{e}^{*}}$ & $2,29^{\mathrm{e}}$ \\
\hline $\begin{array}{l}\text { Fertilizante nitrogenado } \\
\text { (control relativo) }\end{array}$ & $6,28^{\mathrm{d}}$ & $2,60^{\mathrm{d}}$ \\
\hline Azotobacter $\mathrm{sp}$ & $8,54^{\mathrm{c}}$ & $5,78^{\mathrm{c}}$ \\
\hline Burkholderia $\mathrm{sp}$ & $9,99^{\mathrm{b}}$ & $6,00^{\mathrm{b}}$ \\
\hline $\begin{array}{l}\text { Azotobacter } \mathrm{sp} y \\
\text { Burkholderia } \mathrm{sp}\end{array}$ & $10,35^{\mathrm{a}}$ & $7,03^{\mathrm{a}}$ \\
\hline $\begin{array}{l}\text { *Valores con diferentes letras con } \\
\text { estadística (p<0,05) según Tukey. }\end{array}$ & diferencia \\
\hline
\end{tabular}

Lo anterior sugiere que Azotobacter sp. y Burkholderia sp. fueron benéficas para el maíz por una interacción sinérgica entre ambos géneros en favor de su sano crecimiento, incluso mejor que el maíz sin inocular y alimentado con dosis del FN del 100\% (Camelo et al., 2011).

\section{Conclusiones}

En la producción de maíz el empleo de géneros de BPCV como: Azotobacter sp. y Burkholderia sp. permite reducir y optimizar la dosis de FN sin afectar su sano crecimiento, a la vez de mantener la fertilidad del suelo y no causar contaminación de agua superficial o subterránea, al evitar el exceso de FN. La respuesta positiva del maíz a la inoculación con ambos géneros de BPCV, se debió a la acción que tienen para sintetizar SPCV de tipo complementario, lo que mejoró la capacidad de absorción radical del maíz por el FN, en especial por Burkholderia sp. que colonizó el interior de la raíz y de esa forma aseguro la optimización del FN a pesar de reducirlo al $50 \%$.

\section{Agradecimientos}

Al proyecto 2.7 (2014) de la CIC de la UMSNH, Morelia, Mich., México. Al COECYT, Michoacán, México.

\section{Referencias bibliográficas}

Aguirre-Medina, J.F.; Irizar, G.M.B.; Duran, P.A.; Grageda, C.O.A.; Peña Del Rio, M.A.; Loredo, O.C.I.; Gutierrez, B.A. 2009. Los bofertilizantes microbianos alternativa para la agricultura en México, INIFAPSAGARPA Folleto Técnico No 5. Tuxtla, Chiapas, México.

Arellano, Y.; García, E.; Vázquez-Ramos, J.M. 2008. Auxin-stimulation of DNA synthesis and cell cycle proteins during maize germination. Agrociencia 42: 637-644.

Armenta, A.D.; García, C.; Camacho, J.R.; Apodaca, M.A.; Montoya, L.G.; Nava, E. 2010. Biofertilizantes en el desarrollo agrícola de México. Revista Sociedad, Cultura y Desarrollo Sustentable 6: 51-56.

Camelo, M.; Vera, S.P.; Bonilla, R.R. 2011. Mecanismos de acción de las rizobacterias promotoras del crecimiento vegetal. Revista Ciencia y Tecnología Agropecuaria 12: 159-166.

Escobar, C.; Horna, Y.; Carreño, C.; Mendoza, G. 2011. Caracterización de cepas nativas de Azotobacter spp. y su efecto en el desarrollo de Lycopersicon esculentum Mill. "Tomate" en Lambayeque. Scientia Agropecuaria 2: 39-49.

García-Reyna, M.J.; Plata, G.D.; Cárdenas, N.R.; Farias, R.R.; Sánchez-Yáñez, J.M. 2001. Isolation and effect of inoculation of maize with endophytic bacteria of teocintle. Meeting III on Rhizosphere, Dijon, France.

García-Reyna, M.J.; Caballero, M.J., Cárdenas, N.R.; Farías, R.R; Sánchez-Yáñez, J.M. 2000. Aislamiento y densidad de bacterias endofitas de teocintle. XXV. Congreso Nacional de Microbiología, Mérida, Yucatán, México.

García-González, M.M.; Farías-Rodríguez, R.; PeñaCabriales, J.J.; Sánchez-Yáñez, J.M. 2005. Inoculación del trigo var. Pavón con Azospirillum spp. y Azotobacter beijerinckii. Terra 23: 65-72.

García, J.A.; Castillo, A.; Ramírez, M.E.; Rendón, G.; Larqué, M.U. 2001. Comparación de los procedimientos de Tukey, Duncan, Dunnett, HSU y Bechhofer para selección de medias. Agrociencia 35: 79-86. 
Hernández-Rodríguez, A.; Heydrich-Pérez, M.; Diallo, B.; Jaziri, M.E.; Vandeputte, O.M. 2010. Cell-free culture medium of Burkholderia cepacia improves seed germination and seedling growth in maize (Zea mays) and rice (Oryza sativa). Plant Growth Regulation 60: 191-197.

Martínez, E.; Beltrán, E.; López, J. 2011. La arquitectura radicular del maíz (Zea mays $L$ ). Ciencia Nicolaita 53: 48-60.

Mora, E.; Toro, M. 2007. Estimulación del crecimiento vegetal por Burkholderia cepacia, una cepa nativa de suelos ácidos de sabanas venezolanas. Revista Agronomía Tropical 57: 123-128.

Piromyou, P.; Buranabanyat, B.; Tantasawat, P.; Tittabutr, P.; Boonkerd, N.; Teaumroong, N. 2011. Effect of plant growth promoting rhizobacteria (PGPR) inoculation on microbial community structure in rhizosphere of forage corn cultivated in Thailand. European Journal of Soil Biology 47: 44-54.
Reyes, I.; Valery, A. 2007. Efecto de la fertilidad del suelo sobre la microbiota y la promoción del crecimiento del maíz (Zea mays L.) con Azotobacter spp. Bioagro 19: 117-126.

Sánchez-Yáñez, J.M. 2007. Breve Tratado de Microbiología Agrícola Teoría y Práctica. COSUSTENTA, SA de CV, CIDEM, Instituto de Investigaciones Químico Biológicas/Universidad Michoacana San Nicolás de Hidalgo, ISBN 978-97095424-1-7, Morelia, Mich. México. pp. 130-133, 136138, 153-155.

Wong-Villareal, A.; Caballero-Mellado, J. 2010. Rapid identification of nitrogen fixing and legume noduling Burkholderia species based on PCR 16S RNA species-specific oligonucleotides. Systematic and Applied Microbiology 33: 35-43. 